\title{
Dante-Comics zwischen Kanon- und Populärkultur: Spielformen der Hybridisierung und Strategien der Selbstreferenz
}

\begin{abstract}
Comics used to be the standard example of pulp and popular culture. At the same time there was always a tendency to wrap classics in cheap and easily readable comic paper aimed at the younger or less-educated market. Meanwhile comics take in different forms of literary genres, like comic and graphic novels, which join high and low elements and question simplistic classification.

Comics based on Dante and his Commedia show two main tendencies. One would be the simplification of the complex work for literary beginners. The other would be a subtle reflection of and challenging response to that complexity through specific features of graphic and pictorial media. The latter tendency demonstrates the potential of pictorial and figurative expression at an appropriate level of literary verbalization. A close look at some Dante-comics will demonstrate the vital prospects of visual and figurative narration between literary tradition and new approaches.
\end{abstract}

Keywords: Comics; Literaturbearbeitungen; Hoch- und Populärkultur; Unterhaltungskunst; Komplexitätsreduktion; graphische Erzählung; Intertextualität und Selbstreferentialität; Literatur-Comic; Klassiker und Comic-Adaptionen

\section{Der Comic und die Differenzierung zwischen Hoch- und Populärkultur}

Die Differenzierung zwischen populären und „hohen“, ästhetisch ambitionierten Werken galt lange als maßgebliche Orientierungsgrundlage für Prozesse ästhetischer Bewertung. In diesen Rahmen passten sowohl ein eher bürgerliches Kunstverständnis samt entsprechender Rezeptionskultur als auch innovatorische Ästhetiken. Affinitäten bestanden auch zu kulturkritischen Diskursen (wie exemplarisch die Ästhetik Adornos zeigt): Was dem Geschmack der sogenannten „Masse“ entspricht und als „Massenware“ produziert wird, steht oft zumindest unter dem Verdacht, allenfalls Halb- oder Pseudokunst, Unterhaltungskunst und Konsumartikel zu sein. Die Rezeptionsgeschichte des Comics, des Comicstrips - 
des Heftcomics und später auch der Graphic Novel - war von dem skizzierten Bewertungsmuster in besonderem Maße betroffen. Comics galten lange als Unterhaltungskunst ohne ästhetische Ambition, als auf leichte Konsumierbarkeit angelegte Produkte ohne Anspruch auf die Betrachtung als Kunst, ja sogar als Produkte, die den „guten Geschmack“ verderben. Von reaktionären Kritikern vor allem als jugendgefährdend eingestuft, schien die Comiclektüre der Vermittlung kulturell relevanten Wissens im Wege zu stehen, bestenfalls im Sinne der Zeitverschwendung, schlimmerenfalls als Mittel der Verdummung und Verrohung halber Analphabeten. Und doch gab es schon seit den 1940er Jahren auch solche Comics, die Wissen (etwa über Literatur und Geschichte) zu vermitteln suchten. Die bis heute erfolgreiche „Classics Illustrated“-Reihe ist ein Beispiel; sie stand allerdings im Zeichen einer „Popularisierung“ ihrer Gegenstände durch Komplexitätsreduktion. Der typische CI-Comic bot den Inhalt der dargestellten Text-,Klassiker“ in gegenüber den Textvorlagen gekürzter und reduzierter Form und setzte auf eingängige bunte Bilder im geläufigen, leicht lesbaren Comicstil.

\section{Intertextualität, Interpikturalität, Hybridität}

Im Zuge der in den letzten Jahrzehnten erfolgten Aufwertung des Comics zur allgemein anerkannten Gattung wurden verschiedene Kriterien ästhetischer Beurteilung vorgeschlagen und ausprobiert. Dazu gehören die Qualität, Originalität und Komplexität der Bilder und des Zeichenstils und des jeweiligen Textes, des Plots und der Erzählweise (etwa unter den Aspekten der inneren Konsistenz und des Einfallsreichtums), ferner die Originalität der zeichnerischen, textgestalterischen und die Handlung betreffenden Einfälle - sowie die Vielschichtigkeit und ästhetische Komplexität des Text-Bild-Hybrids Comic bzw. Graphic Novel. Parallel zu Beschreibungsverfahren literarischer Texte und bildkünstlerischer Werke wurden und werden graphische Erzählungen vor allem dann als vielschichtig, komplex und mithin ästhetisch reizvoll wahrgenommen, wenn sie in erkennbaren und deutungsrelevanten Beziehungen zur literarischen respektive zur bildkünstlerischen Tradition stehen. Intertextualität, Interpikturalität, Praktiken des Variierens, Parodierens, Travestierens, Formen des Zitats und der Anspielung sind gerade in jüngerer Zeit in den Blick gerückt, wo es darum ging, Comic-Erzählungen zu analysieren. Damit verbunden sind auch Urteile über ihre ästhetischen Qualitäten (Vgl. Bachmann et al. 2012).

Analoges gilt für die verschiedenen Ebenen und Spielformen von Hybridität. Zum einen ist der Comic ja eine per se hybride Darstellungsform bzw. Gattung, und das spezifische Zusammenspiel von Bildlichem und Sprachlichem, von 
Zeichnungen und Texten, bzw. die comicspezifische Schriftbildlichkeit steht meist im Mittelpunkt historischer und theoretischer Darstellungen zum Comic und seiner Geschichte. ${ }^{1}$ Zum anderen bietet die Beziehung von Comics zur Literaturgeschichte wie zur Geschichte der Bilder, Bildprogramme und bildenden Künste ein besonders breites Spektrum an Hybridisierungsoptionen. In Form von Anspielungen, Paraphrasen, Zitaten und Quasi-Zitaten können in der Comic-Erzählung Anregungen, Elemente, Versatzstücke aus verschiedenen medialen und kulturellen Kontexten miteinander verknüpft werden. Als eine Kunst der (variierenden) Wiederholung (vgl. Frahm 2010) hat gerade der Comic eine enge Affinität zum Kombinatorischen, zur Mischung von Heterogenem auf Motiv- und auf Gestaltungsebene, zur Überlagerung und Verschmelzung von Bildprogrammen, Textformen und Stilniveaus, zur Verknüpfung komischer mit tragischen Elementen, von fiktionalen Erzählungen mit informativen Absichten - kurz: zur Darstellung hybridkultureller Text- und Bildwelten.

\section{Literaturcomics}

Graphische Erzählungen, die auf Werke des literarischen Kanons Bezug nehmen, lassen sich mit Fragen der ästhetischen Wertung auf verschiedenen Ebenen verknüpfen (Vgl. Schmitz-Emans 2012). Dies betrifft erstens (per se) die implizite Bestätigung des kanonischen Rangs der gewählten literarischen Vorlage, zweitens mögliche Erörterungen der Frage, ob und inwiefern die graphische Umsetzung der Vorlage sich in der vergleichenden Gegenüberstellung „,bewährt“, ob sie der Vorlage in welchem Sinn auch immer ,gerecht“ wird, ob sie sich mit dieser im Rahmen ihrer eigenen Darstellungsmittel „messen“ kann (oder sich „blamiert“), sowie drittens die Frage nach der ästhetischen Bewertung der Bilderzählung als solcher, also als Beispiel graphisch-narrativer Kunst. Sinnvoll erscheint mit Blick auf die Vielzahl an Spielformen des Literaturcomics übrigens vor allem eine Differenzierung: zwischen solchen Comics, die (entsprechend dem Konzept der „Classics Illustrated“) Kenntnisse über die Vorlage vermitteln wollen, nicht aber bereits voraussetzen, und solchen, die eine Kenntnis der Vorlage oder doch zumindest ein Wissen über diese durchaus voraussetzen. Nur im letzteren Fall erscheinen ästhetische Strategien wie Parodie, Travestie und Pastiche sinnvoll, und viele Literaturcomics bedienen sich gerade dieser Strategien - teils in den Spuren von per se parodierenden oder travestierenden literarischen Genres bzw. angeregt durch literarische Pastiches.

1 Vgl. dazu diverse Beiträge aus Stein und Thon 2013. 
Einen selbstreferenziellen Zug haben Literaturcomics zu erzählenden Texten schon dadurch, dass sie sich implizit (manchmal explizit) in der Tradition literarischen Erzählens verorten. Dies geschieht allerdings unter sehr verschiedenen Akzentuierungen.

Die im Folgenden vorzustellenden Dante-Comics lassen sich entsprechend gruppieren: In Comics für Leser ohne Dante-Wissen und in solche mit entsprechendem Wissen, und sei es einem rudimentären, auf dessen Basis Anspielungen dechiffriert, Zitate erkannt, Parodien verstanden werden. Die Voraussetzungen für letzteres Wissen sind dabei insgesamt in Italien günstiger als in anderen Ländern.

\section{Dante-Comics - Voraussetzungen und Überblick}

Dantes Commedia ist unter dem Aspekt der Adaptierbarkeit durch graphische Erzählungen ein instruktiver Spezialfall. Erstens ist Dante einer der in dieser Rolle völlig unbestrittenen Kanon-Autoren der abendländischen Literatur - und eine graphisch-narrative Umsetzung damit ein Musterfall der Auseinandersetzung mit „Kanonischem“. Zweitens ist der Text der Commedia stark visuell geprägt und lädt daher zur Bebilderung ein. ${ }^{2}$ Drittens lädt die Form der Commedia als Abfolge von Episoden dazu ein, in sequentielle bildliche Darstellungen übersetzt zu werden (Vgl. Hölter und Hölter 2017, 345). Viertens besteht seit Jahrhunderten eine enge Verbindung zwischen Textrezeption und Bebilderungsgeschichte der Commedia. Immer wieder haben Künstler Bilder zur Commedia geschaffen, was sich auf die Rezeptionsgeschichte des Werks selbst nachhaltig ausgewirkt hat. ${ }^{3}$ Comiczeichner stehen daher nicht nur in einer großen Tradition, sie können auch aus reichen Beständen zitieren. Die wichtigsten Stationen der Bebilderungsgeschichte der Commedia - zu deren Hauptgebieten illuminierte Handschriften, Druckgraphien und Gemälde, Buchillustrationen und eigenständige bildkünstlerische Werke gehören - sind zumindest innerhalb der westlichen Kultur weithin bekannt. Dazu gehört die Bildserie Sandro Botticellis (die im Einzelnen bereits auf den Comic vorausweist ${ }^{4}$ ), dazu gehören die visuellen Dante-Interpretationen William Blakes (der nicht nur visuell ganz eigene Akzentuierungen vornimmt, sondern sich auch durch seinen eigenwilligen Umgang mit der Textvorlage ausweist); dazu gehören

2 Hölter und Hölter 2012 (18) sprechen von der „Anschaulichkeit, d. h. der visuellen Suggestionskraft seiner Textphantasie“.

3 Vgl. zu Botticelli Rehm 2017.

4 Botticellis Dante-Illustrationen zeigen teilweise - analog zu den Seiten von Comic-Heften simultan mehrere Phasen einer Geschichte oder Handlungsfolge. 
die Umrissbildzeichnungen von John Flaxmann (dessen Text-Bild-Kombinationen als Vorläufer des Comics betrachtet werden können). Eine besonders bekannte, vielfach zitierte und auch für Comiczeichner besonders wichtige Inspirationsquelle ist der Zyklus mit Commedia-Illustrationen von Gustave Doré. Aber auch bekannte Tafelbilder (etwa von Anselm von Feuerbach und Eugène Delacroix) gehören zu den weithin bekannten Gegenständen des kulturellen Wissens. Insgesamt sind die visuellen Interpretationen der Commedia durch Zeichner, Maler, Graphiker wohl breiter bekannt als die (ebenfalls zahlreichen und oft in Kanonlisten aufgenommenen) Produkte der literarischen Dante-Rezeption. In ihrer Summe bilden sie einen facetten- und spannungsreichen, durchaus als ,hybrid“ zu bezeichnenden Bestand. Als ein Bindeglied, das zwischen Botticellis Bildern zur Commedia und den Dante-Comics neuerer Zeit situiert ist, charakterisieren Achim und Eva Hölter den Zyklus „Dante historiato“ von Federico Zuccari (1586), bei dem die Bilder bereits durch Texte (mit einem „deutlich sekundären Charakter“ [Hölter und Hölter 2017, 345]) ergänzt erscheinen. Spätestens mit der Literatur des 20. Jahrhunderts wendet sich das besondere Interesse der kreativen Rezipienten dem „Inferno“ zu. Auch im Bereich bildkünstlerischer Dante-Rezeption kann wohl von einer tendenziellen Privilegierung des Infernos respektive der Infernobilder gesprochen werden. Dies gilt auch mit Blick auf Dante-Comics.

Diese schließen die Commedia dabei auf unterschiedliche Weisen an die Welt und den Verständnishorizont ihrer Rezipienten an (Vgl. Kretzschmar 2013). $\mathrm{Zu}$ unterscheiden sind entsprechend verschiedene Formen des Brückenschlags zwischen dem spätmittelalterlichen Text auf der einen, dem Lebensumfeld und Wissenshorizont des gegenwärtigen Lesers auf der anderen Seite. So kann es darum gehen, Dantes Text dem zeitgenössischen Leser näherzubringen. Oder darum, auf der Basis dieses Textes eine eigene, möglichst effektvolle Geschichte zu konstruieren. Oder darum, durch die parodistische Verulkung eines kanonisierten Klassikers humoristische Wirkungen zu erzeugen. Oder darum, an Leseund sonstige Lebenspraktiken des Lesers anzuschließen, ein kulturelles Erbe auf unkonventionelle Weise zu pflegen - oder Danteske Gestaltungselemente ornamental oder als Spin-offs anderer Produkte zu verwenden.

Besondere Anschlussstellen für Comic-Adaptationen bieten insbesondere das codifizierte Aussehen des Helden Dante, seine Ähnlichkeit mit „Superhelden“ und die Serialität der Commedia-Episoden. ${ }^{5}$ Einen Überblick über Geschichte und Spielformen des Dante-Comics hat im Jahr 2004 eine Ausstellung in Ravenna

5 Vgl. Hölter und Hölter 2012, 19. Dante ist nicht nur Autor der Commedia, sondern auch als Figur im Text präsent, darum ,weiß‘ man, wie die Figur aussieht - unter Orientierung an Danteporträts. (Wobei die Frage nach deren Authentizität auf einem anderen Blatt steht.) Dante ist zudem als Jenseitsreisender ein „Superheld“, so Hölter und Hölter 2012, 19. 
gegeben (Vgl. Guiducci und Cantarelli 2004). Auch eine im selben Jahr in Erlangen gezeigte Ausstellung über Dante in der bildenden Kunst bezog Comicbeispiele ein. ${ }^{6}$ Darauf aufbauend, haben Achim Hölter und Eva Hölter in einer Abhandlung von 2012 einen panoramatischen Überblick vorgelegt, wichtige Analyseoptionen deutlich gemacht und differenzierte Befunde erhoben. ${ }^{7}$ Dieses Panorama wurde 2017 durch weitere Beispiele und Beobachtungen ergänzt (vgl. Hölter und Hölter 2017). Aber auch diverse andere Studien zu einzelnen Dantecomics und zu allgemeinen Tendenzen haben die sich zunehmend stärker akademisch etablierende Comicforschung bereichert. ${ }^{8}$

Es gibt keine Commedia-Version der „Classics Illustrated“ (Hölter und Hölter 2012, 29), wohl allerdings eine stilistisch ähnliche Dante-Biographie: das Heft „Vita di Dante“, erschienen in der Jugendzeitschrift Il Giornalino (vgl. dazu Guiducci und Cantarelli 2004 sowie Hölter und Hölter 2012, 29). Auffällig ist insgesamt die Vielzahl eher komischer Bilderzählungen und humoristischer Commedia-Adaptationen im Comicstil. Dies zeigt schon eine kurze Übersicht zu einigen älteren Dantecomics. ${ }^{9}$ Hier werden Danteske Motive und Episoden wiederholt mit Bild- und Figurenwelten hybridisiert, die den Comiclesern aus bekannten Reihen vertraut sind.

$\mathrm{Zu}$ den komischen Commedia-Adaptationen gehören $\mathrm{u}$.a. die im DonaldDuck-Stil gezeichneten Geschichten „L'inferno di Topolino“ (in Martina und Bioletto 2001; dazu Hölter und Hölter 2012, 30), ${ }^{10}$ „Paolino Pocatesta e la bella Franceschina“, ${ }^{11}$ „Die schöne Francesca“12 und die „Topolino Story““13. Humoris-

6 Himmel und Hölle: Dantes Göttliche Komödie in der modernen Kunst. Hg. Stadtmuseum Erlangen. Erlangen 2004.

7 Hölter und Hölter (2012) geben einen Überblick über Dantecomics bis 2012, kommentieren Beispiele und skizzieren Tendenzen. Zur Dantecomicproduktion insgesamt: Hölter und Hölter 2012, 18f., unter Bezugnahme auf den Katalog Ravenna 2004: „In der Summe zeigt sich eine gewisse übergreifende Tendenz zum schlichten Historisieren, zur Verschiebung ins Komische oder zum für die Zeichenkunst bequemen Abschweifen ins Visionär-Phantastische.“

8 Vgl. die rezenten Publikationen von Bosold-DasGupta 2010, Muth und Simonis 2013, Kretschmar 2013 und Gröne 2015.

9 Zu den komischen Beispielen vgl. Hölter und Hölter 2012, 30-37.

10 Es agieren vertraute Disney-Figuren (Micky Maus, Donald Duck, Goofy, Pluto, Tick, Trick und Track, Dumbo etc.). Zur Gesamteinschätzung vgl. Hölter und Hölter 2012, 33 f. „Die Grundidee der Disney-Adaptionen ist die Travestie. Wir dürfen also keinen Moment erwarten, eine adäquate Inszenierung des ,Inferno’ zu sehen, sondern lesen Dante im Modus eines heroisch-komischen Epos (...).“

11 In: Topolino, 27.1.1980; Zeichner: Giovan Battista Carpi; dt. Fassung im Lustigen Taschenbuch 88 (1983); Angabe nach Hölter und Hölter 2012, 41.

12 In: Lustiges Taschenbuch 88 (1983), 178-193; Angabe nach Hölter und Hölter 2012, 41.

13 A cura di Gianni Bono. Vol. 1, Milano 2005, 14-16; Angabe nach Hölter und Hölter 2012, 41. 
tisch geprägt ist auch Hunt Emersons Comic „Dante’s Inferno“, 2012 als Album erschienen (dazu Hölter und Hölter 2017, 348). Stilistisch damit stark kontrastierende Adaptationen gestalten die Dante-Welt als zeitgenössische Alltagswelt (so etwa Bach 1973) ${ }^{14}$ oder als Horrorszenario (vgl. den Heft-Comic von Claremont et al. 1980; Angaben nach Hölter und Hölter 2012, 39). Im Folgenden ein Blick auf ausgewählte Beispiele.

\section{Joe Lee: Dante for Beginners. New York und London 2001}

Joe Lees Dante-Einführung gehört zu einer Publikationsserie aus Einführungscomics zu verschiedenen Wissensgebieten (u. a. zu literarischen Gegenständen), ist dabei aber stilistisch nicht einmal ein typisches Comic-Buch. Die Seiten sind nicht als Comic-Panels aus Einzelbildersequenzen strukturiert, sondern folgen einem anderen Schema: Ein relativ großer Textanteil wird durch eine Linie umrahmt; die Zeichnungen wirken mit an der Rahmung des Textes und ragen oft von mehreren Seiten in diesen hinein. Die meisten von ihnen stellen Figuren dar, und hier wiederum die meisten Dante. Würden dabei nicht gelegentlich Sprechblasen verwendet, hätte Lees Buch wenig Ähnlichkeit mit einem Comic.

Der Text, ein zusammenhängender Essay, der wie die Zeichnungen von Lee stammt, dominiert das Buch; die Bilder haben zum einen illustrative Funktion, zum anderen ironisieren sie den Text gelegentlich durch Elemente von Komik; dabei erzählen sie aber keine eigene Geschichte, sondern zeichnen Stationen von Dante Alighieris Leben nach. Lees Text und seine Bilder berücksichtigen auch den zeitgeschichtlichen Hintergrund; die Narration ist mit historischen, kulturhistorischen und soziologischen Informationen verbunden, die in unterhaltendem Stil vorgetragen werden. In diesen Rahmen integriert ist eine zusammenfassende Darstellung der Commedia; diese nimmt rund 80 Prozent des Buchs ein (vgl. Lee 2001, 42-175). Der Reihe nach skizziert werden die Inhalte der einzelnen Canti, zeichnerisch begleitet von einem mit Vergil durch den Danteschen Kosmos reisenden Dante. Lee schreibt in einem umgangssprachlichen Stil, und seine gezeichneten Figuren kommentieren Inhaltliches manchmal mit schnoddrigen Äußerungen,

14 Bach verzichtet auf komisch-parodistische Effekte und verweist stattdessen auf zeitgenössische Themen und Probleme. Wie Hölter und Hölter 2012, 36, treffend konstatieren, ist diese Graphic Novel „ein nur noch lose am Text [Dantes] orientierter gesellschaftskritischer Comic“. Stilistisch signifikant ist u. a. die Verwendung collagierter Photos. 
aber die Information des Lesers hat offenbar Vorrang vor dem Parodistischen. Insofern exponiert sich hier der Comic als ein Erzählmedium, das Unterhaltung und Belehrung verknüpft.

\section{Seymour Chwast: Dante's Divine Comedy. London 2010 (Dt.: Göttliche Komödie. Eine Graphic Novel. München 2011)}

Chwasts Bildergeschichte erzählt die Handlung der gesamten Commedia nach, nicht nur das „Inferno“, wobei allerdings das „Inferno“ den breitesten Raum einnimmt. Am Anfang stehen zwei informative Seiten: eine erste mit einem gezeichneten und aus zwei Textspalten bestehenden Dante-Porträt und allgemeinen Informationen zur Commedia, eine zweite mit einer graphischen Darstellung des Danteschen Weltbildes, das sich an ältere Darstellungen anlehnt; man sieht den Höllentrichter und die Höllenkreise, die zentrale Hölle („Lucifer’s Home“), den Weg hinaus zum „Mt. Purgatory“, der vom „Earthly Paradise“ gekrönt wird (vgl. Muth und Simonis 2013, ferner Hölter und Hölter 2017, 347 f.). Die Erlebnisse der Danteschen Hauptfigur auf ihrer Jenseitsreise werden hier als eine Art „Jedermann“-Geschichte nacherzählt. Chwasts Zeichenstil ist lakonisch und kunstvoll-einfach: In Schwarz-Weiß gehalten, bietet er (partiell schwarz ausgefüllte) Umrisszeichnungen von stilisierten Figuren und stilisierten Ambientes. Diese zeigen, passend zum Sujet, manch Phantastisches (wie Teufel und Ungeheuer), enthalten aber auch viele Elemente der Alltagskultur. Dantesches Inferno, Alltagswelt und Bilder aus dem Bereich der kollektiven Imagination erscheinen überblendet. Dante tritt im Trenchcoat, mit dunkler Brille und Hut auf; ständig die Pfeife im Mund tragend und mit ausgeprägter Nase, erinnert er an typenhafte Detektivfiguren. ${ }^{15}$ Vergil ist das ebenso typenhafte Pendant zu Dantes Erscheinung: Er trägt einen frackartigen Anzug, schwarz-weiße Schuhe, einen Spazierstock und einen Bowler Hat; eher korpulent und mit schmalem Oberlippenbart, ähnelt er typisierenden Darstellungen von Geschäftsleuten. Beatrice trägt ein Kostüm, das ähnlich wie die Kleidung Dantes und Vergils die 1920er Jahre asso-

15 Während all seiner Abenteuer nimmt Dante nie seinen Hut ab, allenfalls angesichts der Aussicht auf eine Dichterkrönung, die ihm eine andere Kopfbedeckung eintragen würde (vgl. Chwast 2010, 118), sowie zuletzt, in Gesellschaft von „St. Bernard“ und der „Virgin Mary“ (vgl. Chwast 2010, 126). Die Pfeife behält er aber auch hier noch im Mund. Und bei der abschließenden Betrachtung des Sternenhimmels hat er auch den Hut wieder auf (vgl. Chwast 2010, 127). 
ziieren lässt; Ähnliches gilt für Lucia. Reminiszenzen an die Welt der 1920er Jahre prägen die Szenerie insgesamt stark; so treten St. James, St. John und St. Peter als Tänzer im Stil damaliger Shows auf. Gerade solche vom Plot selbst keineswegs motivierte Anachronismen, u. a. auch die einer älteren Mode angehörende Kleidung der Figuren, suggeriert, die Bilderzählung sei der geläufigen Zeitordnung nicht unterworfen: Das Dargestellte könnte zu jeder Zeit spielen. Entsprechend bewegen sich auch Menschen, Höllen- und Himmelsbewohner durch dieselbe Welt - wie bei Dante.

Auf der Textebene finden sich knappe Nacherzählungen von Dantes Jenseitsabenteuern, meist in Form narrativer Kommentare, teils in Gestalt von Sprechblasen. Oft werden die Bildmotive auch nur betitelt, Figuren und Orte etikettiert oder mit knappen Bildlegenden versehen. Anflüge von Komik stellen sich ein, wenn Sprachbilder visuell getreu umgesetzt werden, etwa indem Chwast Dante von Lucia zum Tor des Purgatoriums tragen lässt: Man sieht eine elegant gekleidete Frau, die den ,Detektiv، Dante mit sich herumschleppt (vgl. Chwast 2010 72). Auf dezente Weise humoristisch wirkt aber vor allem die Kontrastierung von Danteskem Inhalt und modernen Gestalten, die Bevölkerung der drei Jenseitsreiche mit Figuren in moderner (wenn auch unmodischer) Alltagskleidung, die Arrangements von einerseits zeitspezifischem, andererseits in die ,Ewigkeit“ versetztem Motivmaterial. Chwasts lakonisch-stilisierender Zeichenstil hat seine eigene verhaltene Komik, aber um eine Verulkung von Dantes Commedia geht es ihm nicht, sondern vielmehr um die Effekte, welche sich aus der Hybridisierung von Commedia- und von Alltagsmotiven ziehen lassen, von mittelalterlicher Imagination und Bildprogrammen einer leicht angestaubten Moderne. Als selbstreferenziell zu beschreiben wäre Chwasts Comic vor allem durch die programmatische Einebnung zeitlicher und kultureller Differenzen zwischen Dantes Zeit und dem 20. Jahrhundert: Der Comic, so signalisiert er, erzählt zeitlose Geschichten.

\section{Michael Meier: Das Inferno. Kassel 2012}

Meiers Graphic Novel - die zuerst als täglicher Comicstrip in der Frankfurter Rundschau erschien - erzählt die Abenteuer einer Figur, die sich Dante nennt, ansonsten aber mit dem Dante der Commedia wenig gemeinsam hat (Vgl. Hölter und Hölter 2017, 348 f.). Meiers Dante ist ein sportlicher Typ mit einem Repertoire von allerlei flotten Sprüchen. Auf einer nicht weiter begründeten Gebirgswandertour gerät Dante in einen unübersichtlichen Wald, wo er - analog zu der ersten Episode der Commedia - zum einen mehreren merkwürdigen und bedrohlichen Gestalten begegnet (einer Riesenfrau im Leopardenkleid, einem weitgehend 
anthropomorphen sprechenden Löwen und einem sprechenden Wolf), zum anderen einem Helfer, der ihn aus der vom Wolf drohenden Gefahr rettet und sich als weiterer Begleiter anbietet. Es handelt sich (wie man später auch erklärt bekommt) um einen roten Schakal. Er stellt sich Dante als einstigen Dichter aus Mantua vor, ist also offenbar ein äußerlich verwandelter Vergil. Seine Rolle entspricht im Folgenden auch der Vergils in der Commedia. Die drei unheimlichen Wesen im Wald sind Dantes allegorischen Tieren nachgestaltet: als sinnlich-träge Frau, als psychologisierender und dabei latent gewalttätiger Löwe, als tückischer Versicherungsmakler in Wolfsgestalt repräsentieren sie ,Laster', die den von Dante thematisierten ähneln. Vergil berichtet Dante auch bei Meier von seiner Mission im Auftrag Beatrices, die ihn in seiner Erscheinungsform als Dichter-Büste kontaktiert hatte (vgl. Meier 2012, 14/15). Der zusammen mit dem tiergestaltigen Vergil eingeschlagene Weg der beiden Hauptfiguren führt explizit „durch die Hölle“ (Meier 2012, 12). Bald nach ihrem Aufbruch stehen die Wanderer vor dem Eingang zur Hölle, der die aus der Commedia bekannte Inschrift trägt (,... tu, der du eintrittst, alle Hoffnung ab“ [Meier 2012, 17]). Meiers Graphic Novel gliedert sich in einzelne, durch Zwischenseiten markierte Kapitel, analog der Strukturierung des „Inferno“ in Canti. Auch die Handlung folgt weitgehend der Episodensequenz von Teil I der „Commedia“. Dante und Vergil passieren die Vorhölle und geraten, Höllenflüsse überschreitend wie ihre literarischen Vorbilder, in immer tiefere Kreise der Hölle. Zuletzt gelangen sie, am Teufel vorbei, ins Purgatorio. Die einzelnen Episoden sind durch Anspielungen auf Vorlagen in der Commedia geprägt; Sünder, Höllenstrafen und Erlebnisse Dantes sind parallel zur Vorlage konstruiert, wenn sie auch anders illustriert und ausgesponnen werden, manches fortfällt und manches dazukommt. Ein Unterschied zum groben Handlungsverlauf in der Commedia ergibt sich aber insofern, als Meiers Held sich in der Hölle diversen Verfolgungen und Gefährdungen ausgesetzt sieht, denen er dann jeweils knapp entkommt, obwohl er einiges auszustehen hat. Oft helfen dabei die Erfahrungen und die Gewitztheit des vierbeinigen Vergil.

Meiers Dante-Adaptation arbeitet mit den Mitteln der Travestie: Figuren und Ereignisse der Commedia werden in neuem Gewand re-inszeniert. Für die Höllenszenarien und Höllenstrafen lässt Meier sich einiges einfallen, obwohl man das Muster stets wiedererkennt, gehe es nun um die Episode mit den lebendigen Bäumen (Meier 2012, 58), um den Höllenkreis, in dem die Betrüger sitzen (und wo ein Asiate gefälschte Louis-Vuitton-Taschen verkauft [vgl. Meier 2012, 83]). Minotaurus, der Kentaur, Geryon - alle spielen mit, wenn auch in modifizierter Gestalt. Höllenpersonal und Höllenkulissen erinnern an Horror- und Fantasyszenarien, wobei der lakonisch-flächige Zeichenstil Meiers die Schauereffekte dämpft. Farblich dominieren aber Schwarz und Rot in der ganzen Erzählung. Die Verfremdung der Vorlage ergibt humoristische Effekte, wenngleich es Meier 
auch offenbar nicht darum geht, die Commedia etwa durch Überzeichnung zu verulken. Hinzu kommen die flotte, jargonartige Sprechweise der Figuren sowie auf Komik angelegte Wiederholungen - etwa wenn anlässlich der Begegnung Dantes mit den drei gefährlichen ,Tieren“ im Wald jedes Mal auch Berlusconi auftritt. Die „Modernisierung“ der Höllenstrafen bringt manch komischen Effekt mit sich, etwa wenn auf das Anschauen von Kochshows als schreckliche Strafe angespielt wird oder Berlusconi als ein an verschiedensten Ecken plötzlich auftauchender Medienteufel erscheint. Dabei werden auf satirisch-parodistische Weise auch ,ernsthafte، Probleme zum Gegenstand der Anspielung: brutale Nazis, Alkoholismus, Umweltzerstörung durch Industrie, dubiose Geschäftspraktiken; man trifft auf zänkische Hartz-IV-Empfänger, wird konfrontiert mit einer nervtötenden Bürokratie, mit Atommüll-Lagern, mit Hitler und anderen „Schlächtern“ (Meier 2012, 54).

Meier setzt mehrfache Hybridisierungsstrategien ein. Integriert sind der Geschichte diverse Bildzitate, so eines der Madonna, die mit Beatrice ihre Sorge um Dante teilt (vgl. Meier 2012, 15), ein Bild von Paolo und Francesca (nach einer Vorlage von Ary Scheffer, 1835; vgl. Meier 2012, 26), ein richtender Minos (nach Gustave Doré; vgl. Meier 2012, 26). Gelegentlich wird zur Charakteristik von Phantasien und Gedankenspielen der Hauptfigur Dante mit unterschiedlichen Zeichencodes und -stilen gespielt. So führt eine temporäre Verwirrung Dantes dazu, dass sich einige Panels im unbeholfenen Stil von Kinderzeichnungen präsentieren, eines auch als Abbild von Legosteinen (vgl. Meier 2012, 22). Einen selbstreferenziellen Zug hat Meiers Version der Commedia nicht zuletzt dadurch, dass er den Leser durch einen „Wald“ der Zeichen schickt, der durchdrungen und entziffert werden muss. Das mit vielen Herausforderungen verbundene LeseAbenteuer angesichts eines Comics, der aus ganz unterschiedlichen Registern schöpft, erscheint als humoristisches Äquivalent der mühsamen Reise Dantes, weniger gefährlich, aber ebenfalls spannungsreich.

\section{Joseph Lanzara: Dante's Inferno. The Graphic Novel. New Jersey 2010}

Lanzaras Dante-Comic stellt insofern ein Kuriosum dar, als die Bilder der Bildgeschichte dem Illustrationszyklus Gustave Dorés zur Commedia entnommen sind. ${ }^{16}$ Mittels der Doréschen Bilder erzählt der Comic die Commedia-Handlung

16 „Story: Dante Aleghieri (sic) / Art Gustave Doré“ (innere Titelseite). 
in großen Zügen und als Paraphrase diverser Episoden nach. Allerdings werden neue Akzente gesetzt, so durch Suggestion einer „Schuld“ Dantes - er hat, wie er Vergil erzählt, Beatrice geliebt, Gott hat sie ihm genommen, und daraufhin hat er sich von Gott abgewandt (vgl. Lanzara 2010, 13) - und die Verknüpfung der in der Original-Commedia erzählten Geschichte mit einem von Lanzara erfundenen Handlungsstrang: Gegen Ende der Geschichte, nachdem der Protagonist von Vergil an Beatrice übergeben worden ist, empfindet dieser die Verbannung Vergils aus dem Paradies als so schmerzlich, dass er Beatrice zu einem Fehlverhalten verleitet: Sie ,tauft“ den ob seines Ungetauftseins diskriminierten Vergil mit dem Wasser des Styxs und zieht damit Gottes Zorn auf sich und auf Dante (vgl. Lanzara 2010, 24-25). Zwar entführt ein Adler Beatrice (vgl. Lanzara 2010, 26), aber ebenso unerwartet, wie Dante hier ,bestraft' wird, tritt auch wieder eine positive Wendung ein- mit Verzeihung und Erlösung. Dante erhält Ermahnungen von der Mutter Gottes, die ihn begnadigt (vgl. Lanzara 2010, 29) - und die Hauptfiguren können zusammen das Paradies betrachten - wie bei Doré. In die Doréschen Bilder sind Sprechblasen einmontiert; hinzu kommen gelegentliche Untertitelungen einzelner Panels. Manchmal werden Bilder aus verschiedenen Bildvorlagen Dorés zusammengeschnitten. Dies alles und die Aufteilung der Seiten in (oft halbe, manchmal ganze Seiten füllende) Panels verleiht der Bildgeschichte Montagecharakter. Das stilistisch stark abweichende Cover des Heftes zeigt eine ältere skulpturale Darstellung von Sündern im Fegefeuer, wie man sie aus katholischen Kirchen und Klöstern kennt. Es weckt, zumal in seiner blutroten Rahmung, andere Erwartungen als die von der schwarz-weißen Bildgeschichte dann eingelösten.

Lanzara erzeugt einen eigentümlichen Kontrasteffekt durch die Verknüpfung der Doréschen Dante-Illustrationen mit Sprechblasen - aber parodistisch ist seine Bildgeschichte weder mit Bezug auf Doré noch mit Bezug auf Dantes Text selbst. Auch die Modifikation der Handlung durch die reichlich krude Story um Vergils Taufe ist nicht parodistisch gemeint; Humor liegt der Bildgeschichte überhaupt völlig fern. Man könnte Lanzaras „Inferno“ als eine pastichartige Love-and-HorrorStory mit (pseudo-)metaphysischer Rahmenkonstruktion und ,frommem ' Schluss beschreiben, deren Verfasser Dante zwar keineswegs despektierlich behandelt, sich um den ursprünglichen Sinn der Commedia-Motive und -Episoden aber auch nicht wirklich schert. Der Comic exponiert sich hier selbst als ein in etwas seltsamem Sinn ,belehrendes‘ und pathetisches Medium. 


\section{Christos Gage (Text) / Diego Latorre (Bilder) / Alessandro Benedetti (Lettering): Dante's Inferno. Der Comic zum Hit-Videogame von Electronic Arts. Stuttgart 2010}

In dieser Comicerzählung kommt es zu einer Art Absorption des Dante-Plots durch Plot, Bildprogramm und Logik des gleichnamigen PC-Spiels. Die Handlung ist an die des Spiels angelehnt, die Figuren entsprechend profiliert. Mit den Figuren der Danteschen Commedia haben sie nicht mehr viel zu tun. Aus dem Dichter Dante ist ein Kreuzritter geworden, der sich auf der Suche nach seiner vom Teufel gefangengehaltenen Beatrice in die Hölle begibt - eine vage Reminiszenz an den Mythos um Orpheus. Was im Comic geschieht, sind recht krude Dinge um ein reichlich trivialisiertes und mit Höllenbildern zusammengeschnittenes Mittelalter. ${ }^{17}$ Dante muss allerlei einstecken. Am Ende hat er Beatrice und sich aus der Hölle befreit und Luzifer besiegt, wie ihm Beatrice bestätigt (vgl. vorletzte Seite, unpag.). Die Bildsprache Latorres ist expressiv und dabei insgesamt eher malerisch gehalten als am Duktus von Zeichnungen orientiert. In düsteren Szenen von suggestiver Farbigkeit entfaltet sich das phantastische Geschehen um den Weg des Kriegers Dante durch die Hölle. Durchgängig unscharfe Darstellungen von Figuren und Aktionen sowie ein auf die Suggestion von Dynamik zielender Malstil unterstreichen die Beziehung der Bildgeschichte zu einem mit animierten Figuren zu spielenden PC-Spiel, obgleich die Bildsprache des Comics eine andere ist als die des Spiels selbst. Suggerierte „Pinselstriche“ und „Wischbewegungen“ wirken wie Äquivalente der Spiel-Gestik. Der Textanteil tritt an Signifikanz hinter die Bilder deutlich zurück. Er macht das bilddramatisch inszenierte Geschehen zwar verständlich, beschränkt sich aber auf eher knappe Dialoge in Sprechblasen; die Dialoge wirken oft abgerissen; auf narrative Erläuterungen in weiteren Textfeldern wird weitestgehend verzichtet, und rein quantitativ nehmen die Textanteile auch eher spärlichen Raum ein. Es geht mehr um das Durchspielen von Situationen als um eine kohärente Handlung.

17 Aus dem hinteren Umschlagtext: „Nachdem Dante auf dem Kreuzzug im Heiligen Land gegen die Heiden gekämpft hat, muss er bei seiner Ankunft zu Hause erfahren, dass Beatrice, die Frau, die er liebt und heiraten wollte, ermordet worden ist. Dante ist schuld daran, dass Luzifer die Seele des Mädchens gefangen hält, denn er hat sie betrogen und auf diese Art die Katastrophe herbeigeführt. Voller Schmerz, aber zum Äußersten entschlossen begibt Dante sich in die Hölle und beginnt eine gefährliche Reise, um Beatrice zu befreien. Aber welchen Preis wird er dafür bezahlen?“ 
Wie auch für das PC-Spiel, so hat Dantes Commedia für den Comic von Gage und Latorre nur ein frei verarbeitetes Substrat geboten. Immerhin finden sich Reminiszenzen an die Commedia-Handlung: Die Höllenwächter, der Limbus und Beatrice kommen vor - wenngleich stilistisch stark an Horrorfilme und -Spiele angepasst. Strukturelle Verwandtschaften zwischen Commedia und PC-Spiel die Episodenstruktur und das Modell der Eskalation (sukzessiver Abstieg in die Tiefe bzw. Aufstieg über verschiedene Levels) erleichtern die Adaptation des stofflichen Substrats.

\section{Jodi Picoult: The Tenth Circle. New York 2006. (Dt.: Schuldig. Dt. v. Ulrike Wasel und Klaus Timmermann. München und Zürich 2011)}

Ein Kuriosum unter den Dantecomics bietet dieses Beispiel insofern, als hier die Comics in einen Roman integriert sind. Der Roman erzählt von der 14-Jährigen Trixie (eigentlich: „Beatrice“!), der Tochter des freischaffenden Comiczeichners Daniel. Daniel zeichnet für seine Tochter Comics, Superhelden- und Fantasygeschichten, Geschichten mit Personal aus unterschiedlichen Comic-Genres, aber auch aus Reminiszenzen an Dantes Commedia. Eines Tages wird Trixie zum Opfer eines gewaltsamen Übergriffs und erfährt, wie ,höllisch' die Welt sein kann. Auch in Daniels Leben gibt es dunkle Geheimnisse. Die von Daniel gezeichneten Comicseiten mit Dantesken Motiven unterbrechen die Romanhandlung mehrfach. Auch hier wird eine Vater-Tochter-Geschichte erzählt; Protagonisten des Comics sind Duncan und seine Tochter Tracy. Luzifer hat Tracy entführt, und Duncan steigt, begleitet von einer Vergil-Figur, in die Hölle hinab. Sie passieren Zerberus, die Höllenstadt Dis, den Wald der Selbstmörder - und zuletzt findet Duncan Luzifer. Dieser erklärt Duncan die Spielregeln zur Befreiung Tracys, droht jedoch an, diese müsse sterben, wenn Duncan das Spiel verliere. Das Motiv der Befreiung eines weiblichen Opfers aus Luzifers Gewalt verbindet Picoults Roman mit dem PC-Spiel „Dante's Inferno“ und dem darauf basierenden Comic, erinnert aber auch hier zudem an Orpheus und Eurydike im Hades. - Zwischen der Binnengeschichte des Comics im Roman und der Romanhandlung besteht insofern ein Spiegelungsverhältnis, als die Prüfungen Duncans Spiegelungen der von Daniel zu durchlaufenden inneren Prüfungen sind; um Selbsterkenntnis geht es hier wie dort; Duncan muss sich im 10. Kreis der Hölle einem Spiegel aussetzen. 
Rückblickend auf diese Beispiele zeigt sich, dass gerade Comicversionen der Commedia Spielräume für Hybridisierungen bilden, deren Innovationspotential die jeweiligen Comic-Zeichner, -Texter und -Szenaristen im Zeichen recht verschiedener Intentionen erkunden. Neben den comicspezifischen Formen der Hybridisierung von Text- und Bildelementen sind hier Hybridisierungen zwischen modernen bzw. zeitgenössischen Bildwelten und Danteschen Imaginationen besonders prägend - so unterschiedlich sie im Einzelnen ausfallen. Aber auch zwischen verschiedenen Stilebenen und Gattungsmustern ergibt sich ein Spektrum von Komposit- und Übergangsformen. Gerade in der Konfrontation mit der Commedia entdecken und demonstrieren graphische Erzählungen ihre eigenen gestalterischen Potenziale. Für deren Einschätzung erscheint es als besonders signifikant, ob sie den Text der literarischen Vorlage nebst seiner langen Bebilderungsgeschichte ernst nehmen, ihn womöglich reinszenieren wollen, oder ihn als bloßen Anlass gebrauchen, um spektakuläre, meist unterweltliche, Szenerien zu entwerfen. Beide Verfahrensweisen entsprechen einem jeweils spezifischen Selbstverständnis des Comics, der sich einerseits als Erbe und Fortsetzer einer großen erzählerischen Tradition versteht, andererseits teils mutwillig mit den Versatzstücken des Tradierten spielt.

\section{Literaturverzeichnis}

Bach, Franz J. Göttliche Komödie. Comic-Strip frei nach Dante. Basel: bbv, 1973. Chwast, Seymour. Dante's Divine Comedy. London: Bloomsbury Publishing, 2010.

(Dt.: Göttliche Komödie. Eine Graphic Novel. München: Knesebeck, 2011.)

Claremont, Chris, John Romita jr. und Bob McLeod. Nightcrawler's Inferno; X-Men. X-Men Vol 1 (1963-1981), Annual \#4, 1980.

Gage, Christos (Text), Diego Latorre (Bilder) und Alessandro Benedetti (Lettering): Dante's Inferno. Der Comic zum Hit-Videogame von Electronic Arts. Übersetzung Gerlinde Althoff. Stuttgart: Gustav Lübbe, 2010.

Lanzara, Joseph. Dante's Inferno. The Graphic Novel. New Jersey: New Arts Library, 2010.

Lee, Joe. Dante for Beginners. Hanover/New Hampshire: Steerforth Press, 2001.

Martina, Guido, und Angelo Bioletto. „Micky Maus - Das Inferno“. Die göttliche Entenkomödie und andere Streifzüge durch die Weltliteratur. Berlin: Egmont Ehapa, 2001. 241-314.

Meier, Michael. Das Inferno. Kassel: rotopolpress, 2012.

Picoult, Jodi. Schuldig. Dt. v. Ulrike Wasel und Klaus Timmermann. München und Zürich: Piper, 2011. (Orig.: The Tenth Circle. New York, 2006.) 


\section{Forschungsliteratur}

Bachmann, Christian A., Véronique Sina und Lars Banhold (Hg.). Comics intermedial. Beiträge zu einem interdisziplinären Forschungsfeld. Essen: Christian Bachmann, 2012.

Bosold-DasGupta, Bettina. „Dante ,travestito“: Von Eduardo Sanguinetis ,Commedia dell'Inferno' zum Comic“. Dante Alighieri und sein Werk in Literatur, Musik und Kunst bis zur Postmoderne. Hg. Klaus Ley. Tübingen: Francke, 2010. 43-55.

Frahm, Ole. Die Sprache des Comics. Hamburg: Fundus, 2010.

Gröne, Maximilian. „Die Jenseitsreise als Medientransfer: Dantes ,Divina Commedia“ in Comic und Videogame“. Comics - Übersetzungen und Adaptionen. Hg. Nathalie Mälzer. Berlin: Frank \& Timme, 2015. 125-140.

Guiducci, Paolo, und Loris Cantarelli (Hg.). Nel mezzo del cammin di una vignette ... Dante a fumetti. Il sommo poeta e la ,Divina Commedia“ nelle nuvole parlanti di tutto il mondo. Ravenna: Pagina, 2004.

Hölter, Achim, und Eva Hölter. „Dante Alighieri im Comic - eine kurze Aktualisierung“. Komparatistische Perspektiven auf Dantes „Divina Commedia“. Lektüren, Transformationen und Visualisierungen. Hg. Stephanie Heimgartner und Monika Schmitz-Emans. Berlin und Boston: de Gruyter, 2017. 345-352.

Hölter, Achim, und Eva Hölter. „Dante im Comic“. Comic und Literatur: Konstellationen. Hg. Monika Schmitz-Emans. Berlin und Boston: de Gruyter, 2012. 17-49.

Kretzschmar, Karl-Christian. „Neue Dante-Comics“. Il Nuovo Giorno. Mitteilungsblatt der deutschen Dante-Gesellschaft, Juni (2013): 34-39.

Muth, Laura, und Annette Simonis. „Dantes Divina Commedia als Graphic Novel. Seymour Chwasts kurzweilige Dante-Adaption im Comic“. Dante deutsch. Die deutsche Danterezeption im 20. Jahrhundert in Literatur, Philosophie, Künsten und Medien. Hg. Michael Dallapiazza und Annette Simonis (= Jahrbuch für Internationale Germanistik. Reihe A, Bd. 114). Bern et al.: Peter Lang, 2013. 131-148.

Rehm, Ulrich. „Kultivierung des Blicks“. Komparatistische Perspektiven auf Dantes ,Divina Commedia'. Hg. Stephanie Heimgartner und Monika Schmitz-Emans. Berlin und Boston: de Gruyter, 2017. 121-156.

Schmitz-Emans, Monika (in Zusammenarbeit mit Christian Bachmann). Literatur-Comics. Adaptationen und Transformationen der Weltliteratur. Berlin: de Gruyter, 2012.

Stein, Daniel, und Jan Noel Thon (Hg.): From Comic Strips to Graphic Novels. Contributions to the Theory and History of the Graphic Narrative. Berlin und Boston: de Gruyter, 2013.

Monika Schmitz-Emans, seit 1995 Professorin am Lehrstuhl für Allgemeine und Vergleichende Literaturwissenschaft an der Ruhr-Universität Bochum. Forschungsschwerpunkte: Literaturtheorie und Poetik, Literatur und Bilder, Literatur, Graphie und Buchgestaltung. Publikationen u.a. Franz Kafka. Epoche Werk - Wirkung (München 2010); Literatur-Comics. Adaptationen und Transformationen der Weltliteratur (Berlin und Boston 2012, in Zusammenarbeit mit Christian Bachmann). 